\title{
Importancia de la resonancia magnética en el diagnóstico y pronóstico de la cardiomiopatía hipertrófica apical, a propósito de un caso clínico
}

\author{
Importance of magnetic resonance imaging in the diagnosis and prognosis of apical \\ hypertrophic cardiomyopathy, a case report and literature review
}

\author{
Raul Alca-Clares ${ }^{1}$, Jorge Salinas-Arce ${ }^{2 *}$, Henry Anchante-Hernández ${ }^{1}$ y Félix Medina-Palomino ${ }^{1}$ \\ ${ }^{1}$ Servicio de Cardiología, Hospital Cayetano Heredia; ${ }^{2}$ Unidad de Arritmias, Clínica Delgado. Lima, Perú
}

La cardiomiopatía hipertrófica $(\mathrm{CMH})$ es la enfermedad cardiaca hereditaria más frecuente y la segunda causa de muerte cardiaca súbita (MCS) después de la enfermedad coronaria'.

Una de las variantes más infrecuentes es la $\mathrm{CMH}$ apical (CMHA). La estratificación del riesgo de CMHA y el implante de un cardiodesfibrilador (CD) como prevención primaria es una decisión compleja, que en la mayoría de los casos no está incluida en los modelos de estratificación europeo y americano.

Presentamos el caso de una paciente mujer de 41 años, sin antecedentes cardiovasculares y en evaluación prequirúrgica por colecistectomía. No describe palpitaciones ni síncope.

En el electrocardiograma presentó ritmo sinusal, complejos QRS de amplitud incrementada y cambios difusos en la repolarización (Fig. 1A).

En la ecocardiografía transtorácica, el grosor parietal máximo del ventrículo izquierdo (VI) se observó a nivel apical $(21 \mathrm{~mm})$, con extensión al tercio medioventricular (Fig. 1 B y C). La gradiente del tracto de salida del VI se estimó en $8 \mathrm{mmHg}$ y la gradiente medioventricular fue $33 \mathrm{mmHg}$ (Fig. 1D). La deformación global longitudinal (DGL) resultó en $-12 \%$ (Fig. 1E).

De acuerdo con el modelo de estratificación europeo, el riesgo de MSC estimado para los siguientes cinco años fue del $2.06 \%$ (bajo riesgo); según el modelo americano, no se identificaron factores de riesgo convencionales. Ambas herramientas no recomendaron el implante de CD como prevención primaria.

La evaluación con resonancia magnética encontró acinesia apical y obliteración de la cavidad ventricular en sístole (Fig. 2 A y B), presentando realce tardío de gadolinio (RTG) en las zonas de máxima hipertrofia, cuantificado en un $23.9 \%$ (Fig. $2 \mathrm{C}$ ).

El estudio de perfusión miocárdica no mostró isquemia inducible. La región apical presentó un mayor brillo del trazador (Fig. 2D).

Considerando que en nuestro caso las guías clínicas son insuficientes para la estratificación del riesgo y basados en la presencia de una CMHA con aneurisma apical (AA), RTG extenso, obstrucción medioventricular y alteración de la DGL, recomendamos el implante de un $\mathrm{CD}$ y el inicio de anticoagulación.

La CMHA constituye el $4 \%$ de los diferentes fenotipos de $\mathrm{CMH}$ y tiene una mortalidad cardiaca del $0.5 \% /$ año ${ }^{2}$. Sin embargo, ningún esquema de estratificación del riesgo individualiza a este tipo de pacientes.

La asociación con AA, observada hasta en el $20 \%$ de los casos, y la presencia de un fenotipo apical con extensión medioventricular (CMHA tipo B), como lo observado en nuestra paciente, está asociada a un
Correspondencia:

*Jorge Salinas-Arce

E-mail: jsalinas.arritmias@yahoo.com
Disponible en internet: 23-02-2021 (c) 2020 Instituto Nacional de Cardiología Ignacio Chávez. Publicado por Permanyer. Este es un artículo open access bajo la licencia CC BY-NC-ND (http://creativecommons.org/licenses/by-nc-nd/4.0/).

Fecha de recepción: 10-04-2020

Fecha de aceptación: 29-06-2020 DOI: 10.24875/ACM.20000142

\footnotetext{
Arch Cardiol Mex. 2021;91(1):143-146
www.archivoscardiologia.com

Arch Cardiol Mex. 2021;91(1):143-146
www.archivoscardiologia.com
} 


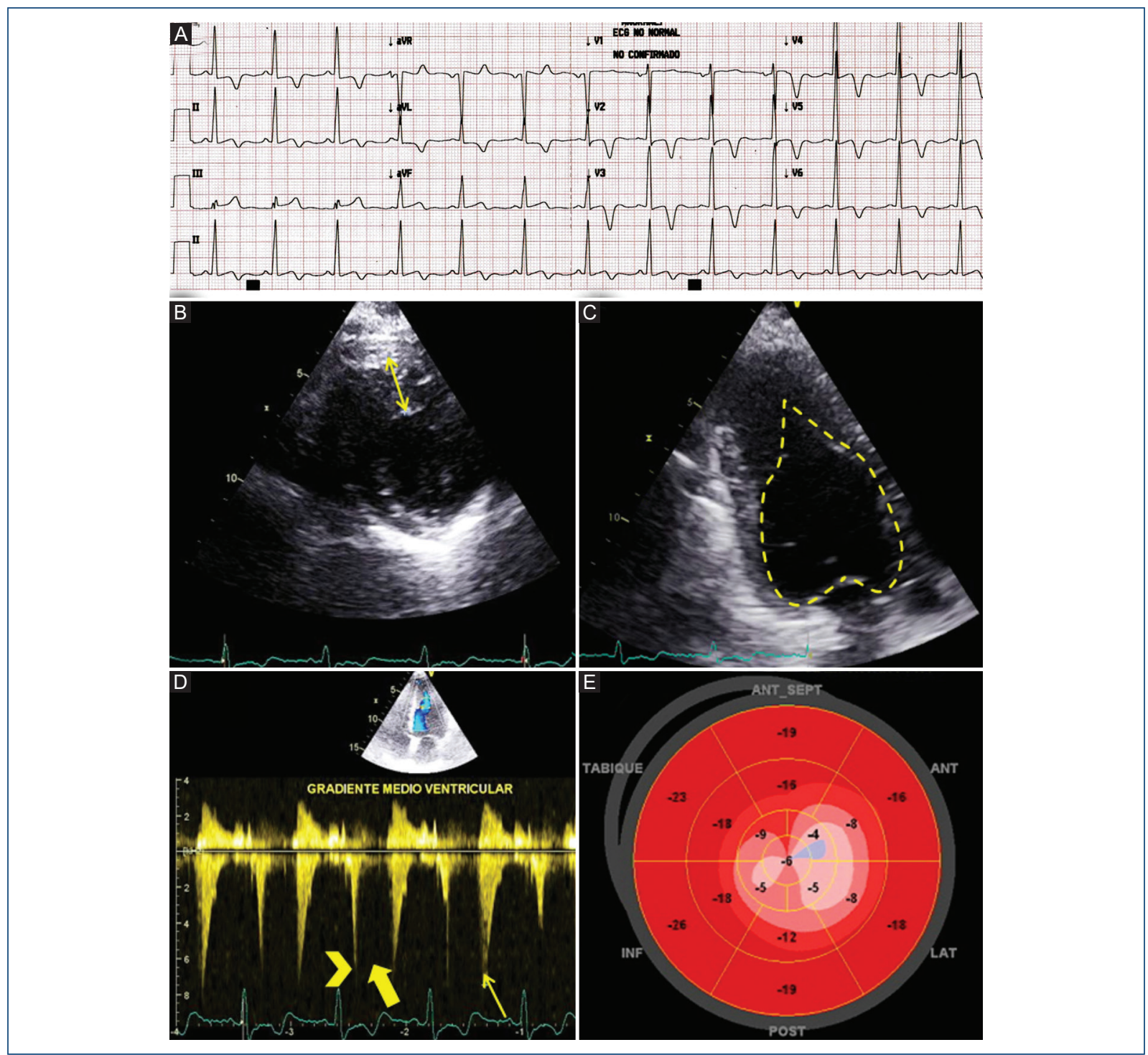

Figura 1. A: electrocardiograma. Onda S profunda en V1 (13 mm) y onda R alta en V5 (28 mm); ondas T negativas (-8 mm en V3) desde V2 a V6, DI y aVL; ausencia de ondas 0 en derivadas laterales; elevación del punto J (1 mm) en DIII y aVF, con patrón rR' en DIII y onda R empastada en aVF. Ecocardiografía. B: eje corto paraesternal izquierdo a nivel apical, grosor máximo telediastólico de $21 \mathrm{~mm}$. C: vista apical de 3 cámaras, se aprecia la cavidad ventricular en forma de «haz de espadas" en telediástole y engrosamiento apical y medioventricular. D: turbulencia medioventricular con gradiente máxima señalada por la cabeza de flecha, seguido de un vacío de señal Doppler en mesosístole (flecha gruesa) y un flujo de jet paradójico en protodiástole (flecha delgada). E: ojo de buey. Alteración segmentaria a predominio apical.

riesgo de MCS tres veces mayor y a una mayor probabilidad de progresión a insuficiencia cardiaca ${ }^{3,4}$.

La evaluación de la $\mathrm{CMH}$ mediante resonancia magnética y la cuantificación del RTG tienen un valor especial en el subgrupo de pacientes definidos como de «riesgo bajo» (sin factores de riesgo convencionales). EI RTG se correlaciona significativamente con eventos de MCS, una extensión de RTG > 15\% se asocia a un riesgo incluso mayor al 6\%/5años ${ }^{5}$. En forma especial, los pacientes con CMHA y AA presentan un incremento significativo del riesgo incluso con la presencia de RTG $>5 \%$.

La incorporación del RTG y la presencia de AA como variables independientes identificó prospectivamente al 95\% de pacientes que desarrollaron arritmias potencialmente letales, previniendo casi todos los casos de MCS?. En la actualidad la resonancia magnética no solo juega un rol en la identificación de los diferentes patrones 


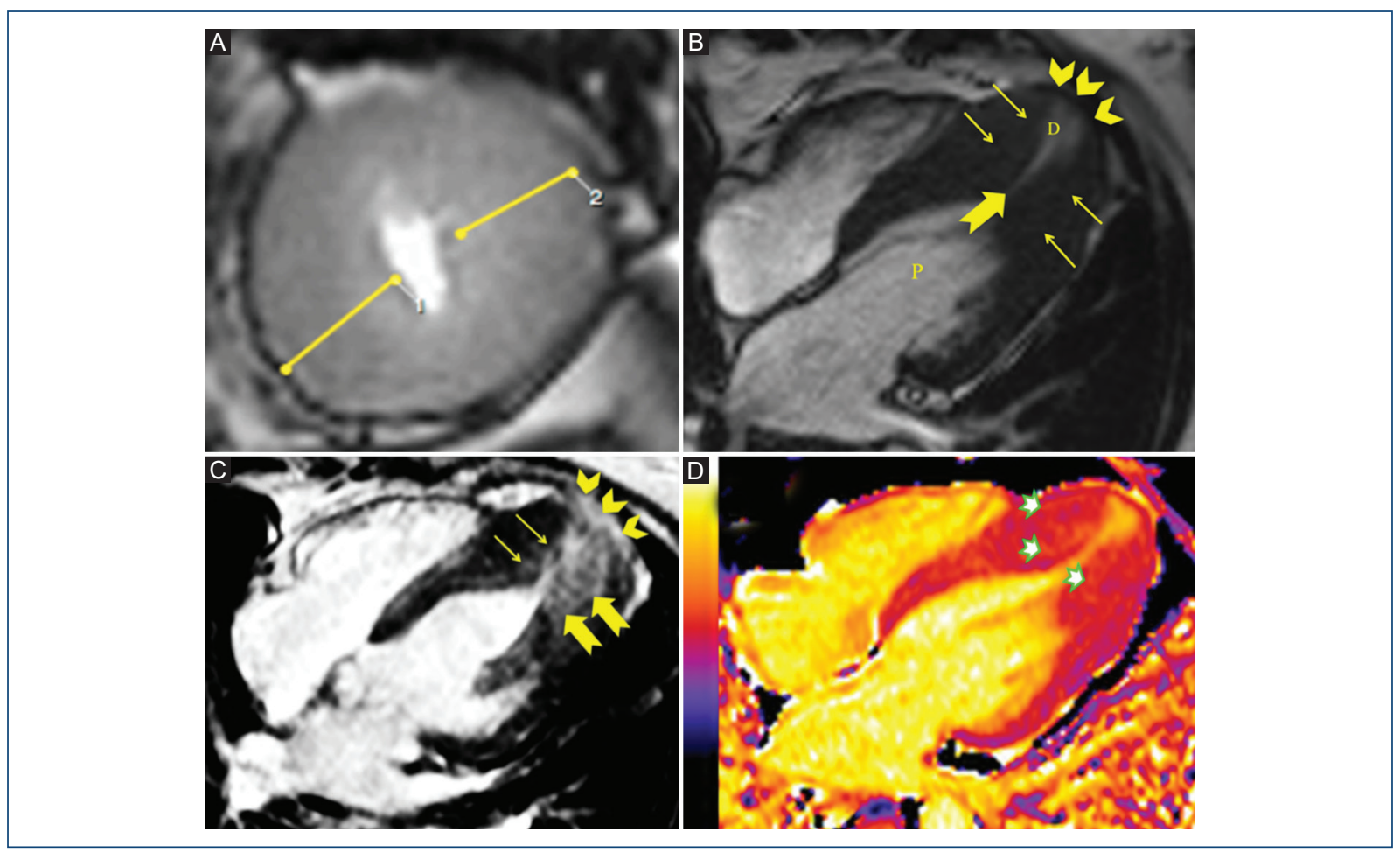

Figura 2. Resonancia magnética cardiaca. A: eje corto apical, grosor parietal máximo en 23 mm. B: vista de cuatro cámaras. Aneurisma apical (cabeza de flechas) de $17 \mathrm{~mm}$, sin presencia de trombo. La hipertrofia apical se extiende hasta la base de los músculos papilares (flechas delgadas) con obstrucción medioventricular por coaptación de la pared anterolateral y septal en sístole (flecha gruesa), lo cual genera una cavidad proximal (P) y otra distal (D). C: realce tardío de gadolinio (RTG). Se observa un patrón de RTG no isquémico parcheado localizado en ápice y borde aneurismático (cabeza de flechas) con extensión a áreas contiguas de la porción distal de pared anterolateral (flechas gruesas) e inferoseptal (flechas delgadas). D: T1 mapping. Incremento de señal hasta valores de 1,077-1,104 ms en zonas de máxima hipertrofia. Los rangos de normalidad fueron entre 960-1,060 ms.

morfológicos de la $\mathrm{CMH}$, sino que además es una herramienta útil en la estratificación del riesgo para muerte súbita, en especial en aquellos pacientes definidos como de «bajo riesgo» según los esquemas de estratificación ${ }^{8}$.

La estratificación del riesgo de los pacientes con CMHA para complicaciones cardiovasculares debe ser realizada en forma individualizada, al constituir un grupo con características especiales que incluyen factores de riesgo aún no reconocidos en las guías actuales.

\section{Financiamiento}

La presente investigación no ha recibido ninguna beca específica de agencias de los sectores público, comercial o sin ánimo de lucro.

\section{Conflictos de intereses}

Los autores declaran no tener conflicto de intereses.

\section{Responsabilidades éticas}

Protección de personas y animales. Los autores declaran que para esta investigación no se han realizado experimentos en seres humanos ni en animales.

Confidencialidad de los datos. Los autores declaran que han seguido los protocolos de su centro de trabajo sobre la publicación de datos de pacientes.

Derecho a la privacidad y consentimiento informado. Los autores declaran que en este artículo no aparecen datos de pacientes.

\section{Bibliografía}

1. Weissler-Snir A, Allan K, Cunningham K, Connelly KA, Lee DS, Spears DA, et al. Hypertrophic cardiomyopathy-related sudden cardiac death in young people in Ontario. Circulation. 2019;140(21):1706-16.

2. Klarich KW, Attenhofer Jost $\mathrm{CH}$, Binder J, Connolly HM, Scott CG, Freeman WK, et al. Risk of death in long-term follow-up of patients with apical hypertrophic cardiomyopathy. Am J Cardiol. 2013;111(12): 1784-91. 
Arch Cardiol Mex. 2021;91(1)

3. Kubo T, Kitaoka H, Okawa M, Hirota T, Hoshikawa E, Hayato K, et al. Clinical profiles of hypertrophic cardiomyopathy with apical phenotypecomparison of pure-apical form and distal-dominant form. Circ $\mathrm{J}$ Off $J$ Jpn Circ Soc. 2009;73(12):2330-6.

4. Yan L-R, Zhao S-H, Wang H-Y, Duan F-J, Wang Z-M, Yang Y-J, et al. Clinical characteristics and prognosis of 60 patients with midventricular obstructive hypertrophic cardiomyopathy. J Cardiovasc Med Hagerstown Md. 2015;16(11):751-60.

5. Chan RH, Maron BJ, Olivotto I, Pencina MJ, Assenza GE, Haas T, et al Prognostic value of quantitative contrast-enhanced cardiovascular magnetic resonance for the evaluation of sudden death risk in patients with hypertrophic cardiomyopathy. Circulation. 2014;130(6):484-95.
6. Hanneman K, Crean AM, Williams L, Moshonov H, James S, Jiménez-Juan $\mathrm{L}$, et al. Cardiac magnetic resonance imaging findings predict major adverse events in apical hypertrophic cardiomyopathy. J Thorac Imaging. 2014;29(6):331-9.

7. Maron MS, Rowin EJ, Wessler BS, Mooney PJ, Fatima A, Patel P, et al. Enhanced American College of Cardiology/American Heart Association Strategy for Prevention of Sudden Cardiac Death in High-Risk Patients With Hypertrophic Cardiomyopathy. JAMA Cardiol. 2019;4(7):644-57.

8. Todiere G, Nugara C, Gentile G, Negri F, Bianco F, Falletta C, et al. Prognostic role of late gadolinium enhancement in patients with hypertrophic cardiomyopathy and low-to-intermediate sudden cardiac death risk score. Am J Cardiol. 2019;124(8):1286-92. 